\title{
Rationale for Early Diagnosis of Mild Cognitive Impairment (MCI) Supported by Emerging Digital Technologies
}

\author{
M.N. Sabbagh ${ }^{1}$, M. Boada ${ }^{2}$, S. Borson ${ }^{3}$, M. Chilukuri ${ }^{4}$, P.M. Doraiswamy ${ }^{5}$, B. Dubois ${ }^{6}$, J. Ingram ${ }^{7}$, A. Iwata In $^{8}$ \\ A.P. Porsteinsson ${ }^{9}$, K.L. Possin ${ }^{10}$, G.D. Rabinovici ${ }^{10}$, B. Vellas ${ }^{11}$, S. Chao ${ }^{12}$, A. Vergallo ${ }^{13}$, H. Hampel He $^{13}$
}

1. Cleveland Clinic Lou Ruvo Center for Brain Health, Las Vegas, NV, USA; 2. Research Center and Memory Clinic, Fundació ACE, Institut Català de Neurociències Aplicades, Universitat Internacional de Catalunya, Barcelona, Spain; and Networking Research Center on Neurodegenerative Diseases (CIBERNED), Instituto de Salud Carlos III, Spain; 3. University of Washington School of Medicine, Seattle, Washington, and Dementia Care Research and Consulting, Santa Ana, CA, USA; 4. Durham Family Medicine, Durham, North Carolina, USA; 5. Departments of Psychiatry and Medicine, Duke University School of Medicine, USA; 6. Institute of Memory and Alzheimer's Disease (IM2A), Department of Neurology, Center of excellence of neurodegenerative disease (CoEN) and National Reference Center for Rare or Early Dementias Pitié-Salpêtrière Hospital, AP-HP, Boulevard de l'hôpital, Paris, France; 7. Seniors Lead Physician, Central East Region, Ontario and Founder and Medical Director of Kawartha Centre, Peterborough, Ontario, Canada; 8. Department of Neurology, The University of Tokyo Graduate School of Medicine, Tokyo, Japan; 9. Department of Psychiatry, University of Rochester School of Medicine and Dentistry, Rochester, NY, USA; 10. Memory \& Aging Center, Departments of Neurology, Radiology and Biomedical Imaging, University of California San Francisco, San Francisco, USA; 11. Gerontopole, Toulouse University Hospital, UMR 1027, University of Toulouse, France; 12. ClearView Healthcare Partners - Newton, MA, USA; 13. Global Medical Affairs, Neurology Business Group, Eisai Inc., Woodcliff Lake, New Jersey, USA

Corresponding Author: Marwan N. Sabbagh, Cleveland Clinic Lou Ruvo Center for Brain Health, Las Vegas, NV, USA, sabbagm@ccf.org; Tel.: (702) 483-6029; Fax: (702) 7226584

J Prev Alz Dis 2020;3(7):158-164

Published online March 6, 2020, http://dx.doi.org/10.14283/jpad.2020.19

\begin{abstract}
Disease-modifying pharmacotherapies for Alzheimer's Disease (AD) are currently in late-stage clinical development; once approved, new healthcare infrastructures and services, including primary healthcare, will be necessary to accommodate a huge demand for early and large-scale detection of AD. The increasing global accessibility of digital consumer electronics has opened up new prospects for early diagnosis and management of mild cognitive impairment (MCI) with particular regard to AD. This new wave of innovation has spurred research in both academia and industry, aimed at developing and validating a new "digital generation" of tools for the assessment of the cognitive performance. In light of this paradigm shift, an international working group (the Global Advisory Group on Future MCI Care Pathways) convened to elaborate on how digital tools may be optimally integrated in screening-diagnostic pathways of AD The working group developed consensus perspectives on new algorithms for largescale screening, detection, and diagnosis of individuals with MCI within primary medical care delivery. In addition, the expert panel addressed operational aspects concerning the implementation of unsupervised at-home testing of cognitive performance. The ultimate intent of the working group's consensus perspectives is to provide guidance to developers of cognitive tests and tools to facilitate the transition toward globally accessible cognitive screening aimed at the early detection, diagnosis, and management of $\mathrm{MCI}$ due to $\mathrm{AD}$.
\end{abstract}

Key words: Alzheimer's disease, mild cognitive impairment, cognitive screening, disease modifying, digital, healthcare.

\section{Introduction}

$\mathbf{T}$ he late-stage clinical development and potential near-approval of drugs with modifying effectd on Alzheimer's disease (AD) calls for a substantial paradigm shift in the diagnosis and Received January 9, 2020 management of the disease, including the Mild Cognitive Impairment (MCI) stage (also called $\mathrm{MCI}$ due to $\mathrm{AD}$ or prodromal AD). The availability of disease-modifying therapies will result in unprecedented demand for cognitive performance assessments (i.e., large-scale cognitive screening). Moreover, the progressive rise of lifespan in populous developed countries such as the U.S., EU5, China, and Japan $(1,2)$ will bring about an exponential increase in the incidence of age-related diseases, including AD,. It is expected that widespread demand for cognitive evaluation will likely overwhelm existing healthcare infrastructures and services at both primary care and specialist levels.

Early detection, of MCI or preclinical AD stages, coupled with timely initiation of disease-modifying treatments, has become the clear path to successfully facing the social and medical threat of AD. Gaps on both sides,particularly a paucity of detection tools suitable for practical use in patient populations and the absence of approved disease-modifying therapies, impede progress toward finding effective therapeutics. We focus here on MCI, a syndrome defined by clinical, cognitive, and functional criteria and characterized by objective cognitive decline in one or more cognitive domains with no significant impairment in daily-life activities (3). MCI may result from a variety of underlying causes, including Alzheimer's pathophysiology $(4,5)$. As a result, watchful monitoring of adults with $\mathrm{MCI}$ is a crucial step within the work-up for early identification of $\mathrm{AD}$ and will be a critical stage of treatment monitoring as novel diseasemodifying $\mathrm{AD}$ therapies enter the marketplace. In this publication series, we use the term "MCI" to refer to non-dementia cognitive impairment due to any cause and the terms "MCI due to AD" or "MCI-AD" to refer specifically to $\mathrm{MCI}$ associated with positive biomarkers 
of $\mathrm{AD}$ pathophysiology, as established in current research diagnostic criteria (3-5).

If disease modifying-therapies enter clinical practice, several issues must be overcome to achieve large-scale cognitive and biological screening of AD.

First, MCI is heterogeneous in its clinical spectrum and has historically been challenging to define, identify, and monitor in clinical practice. In addition, the currently qualified biomarkers for $\mathrm{AD}$ are assessed through invasive, expensive, and time- and resource-consuming investigations such as cerebrospinal fluid (CSF) analysis and positron-emission tomography (PET) imaging. The progressive establishment of blood-based biomarkers (6) and the validation of multi-dimensional diagnostic techniques have the potential to make the diagnosis and management of MCI-AD feasible in primary care, as is necessary for early screening and detection.

Primary care physicians (PCPs) currently lack technical support, infrastructure, training, and experience to efficiently detect and manage $\mathrm{AD}$ along its clinical continuum, from preclinical phases to $\mathrm{MCI}$ and dementia. A 2019 survey conducted among U.S. PCPs reported that short cognitive evaluations are assessed in only half of individuals 65 years of age and older and that cognitive evaluations are frequently omitted due to: i) subtle cognitive impairment, ii) lack of time, and iii) patient resistance to testing (7). In a parallel patient survey, only $16 \%$ of Americans aged 65 years and older reported receiving regular cognitive assessments during routine health visits (7). Less than half of older adults report ever having discussed their cognitive performance with a physician, and less than a third have ever been assessed for cognitive impairment. Additionally, a majority of surveyed PCPs reported uncertainty around which cognitive assessment to deploy, how to perform a brief cognitive assessment, and, importantly, what to do after assessing cognition (7). Referral to specialists (e.g., neurologists, psychiatrists, geriatricians, and neuropsychologists) is the default for evaluating cognition and diagnosing MCI-AD. However, with the expected AD (and other neurodegenerative disease) epidemic burden, access to specialists has been and will become even more challenging. PCPs will need to be fully involved in the process and equipped with the proper means to ensure timely and efficient detection and care.

In recognition of current challenges around the detection of $\mathrm{MCI}$, a working group composed of international experts on $\mathrm{MCI}$ and $\mathrm{AD}$ convened in April 2019 to elaborate on existing frictions and barriers, in both clinical and non-clinical settings, that prevent widespread cognitive screening for early detection of $\mathrm{MCI}$ and particularly MCI-AD. We summarize potential solutions to overcoming those barriers in a series of three manuscripts, of which this is the first. The first manuscript focuses on advantages and disadvantages of early detection of MCI, the current MCI detection landscape, and data-driven hypothetical models on how
MCI-AD diagnosis and management may change in the future given recent technological advances and potential approval of disease-modifying therapies. In the second manuscript of this series, we offer recommendations around ways to meaningfully and rapidly implement MCI detection in primary care settings. The third manuscript of this series will focus on the potential for direct-to-consumer cognitive testing intended for use by adults or informants in an at-home setting without direct supervision by a healthcare provider. Given the critical importance of these topics, the recommendations outlined across this suite of manuscripts reflect careful consideration by this group of cognitive neuroscientists and physicians and extensive iteration and discussion from April 2019 through the present.

From the outset of this endeavor, the primary objective of this working group has been to identify actionable methods to improve detection of $\mathrm{MCI}$, thereby providing developers and researchers of novel tools and tests with guidance and tangible recommendations to maximize their potential usability. To that end, the group agreed that the most feasible strategy to optimize early detection of MCI in the near-term will be to boost primary care capacity for detection by providing infrastructure and equipment that improve the accuracy and efficiency of tools for cognitive assessment without substantially increasing workload for primary care clinicians. Recommendations and strategies to facilitate this paradigm shift are described in detail in the second manuscript of this series.

Our group recognized that detection tools intended for use outside of clinical settings present unique challenges, yet they deserve critical future development, because they offer the potential to dramatically improve the scale of MCI detection. With dramatic increases in the use of consumer electronics by aging adults, digital approaches that leverage the capacities of mobile devices and Internet connectivity are a promising avenue for detection of $\mathrm{MCI}$ in non-clinical settings, if these consumer-directed resources can be suitably validated and linked to healthcare systems. The third manuscript in this series summarizes the challenges and opportunities relating to the detection of $\mathrm{MCI}$ in non-clinical settings, i.e., at an individual's home or in everyday settings such as pharmacies or community screening events, as has already been accomplished with brief paper and pencil tests $(8,9)$.

In this initial manuscript, we summarize existing guidelines and consensus statements to provide context around this set of recommendations. We also weigh the advantages and disadvantages of early detection of MCI, and we ultimately support the idea that detection of MCI is an important component of whole person care. Finally, we summarize our expectations of how the MCI detection landscape may continue to shift in the next $3-5$ years to highlight the need for proactive changes and ongoing research and development. 


\section{Advantages and drawbacks of MCI detection}

Consistent with previous guidelines and consensus statements (10), this panel recognized that early detection of $\mathrm{MCI}$ is associated with both advantages and disadvantages, and we acknowledge that the decision to assess an individual's cognitive function should be made on a case-by-case basis with each individual's best interests in mind. To support large-scale cognitive screening, the field would requirea clinical consensus on the appropriate course of action for PCPs when an individual is identified as cognitively impaired. Additionally, large-scale cognitive screening algorithms were acknowledged as unlikely to become standard practice in the near-term, given the infrastructural challenges inherent to existing healthcare systems around the world. In this context, we have summarized the most critical benefits and drawbacks of early detection of MCI identified by this working group.

\section{Benefits of Early Detection of MCI}

If disease-modifying therapies for delaying or even halting AD at its MCI stage (also called prodromal stage) become available, the necessity of detecting MCI accurately, extensively, and in a timely manner is obvious. Moreover, the inability to robustly identify patients at prodromal stages remains a substantial limitation for developing AD therapies and may, at least in part, contribute to the series of drug failures. Accordingly, early MCI detection may optimize identification of patients eligible for future clinical trials and maximize the likelihood of successfully developing novel AD therapies.

However, even in the absence of a disease-modifying therapy, and regardless of the underlying etiology, multiple advantages remain associated with early detection of MCI. Individuals and healthcare systems can only benefit from an efficient algorithm for investigating $\mathrm{MCI}$ at a large-scale.

An early identification of MCI also increases the possibility of a timely diagnosis of the medical condition that may underlie a cognitive impairment (i.e., secondary cause of $\mathrm{MCI}$ ), which are all potentially treatable or even reversible (e.g., metabolic and endocrine diseases, mood and sleep disorders, iatrogenicity) (11). In addition, growing evidence demonstrates that specific lifestyle habits and activities may slow down or even prevent cognitive decline. Early detection of MCI may provide subjects with greater motivation to implement lifestyle modifications and, at a minimum, will provide physicians with an additional opportunity to counsel individuals on lifestyle changes. In the current screening and diagnostic paradigm, the identification of MCI is likely to escape the therapeutic window where individuals may benefit from these non-pharmacological interventions to slow cognitive decline. Early identification of cognitive impairment also can help individuals and their families better prepare for future care needs and address financial planning considerations, for example. In the absence of a disease-modifying therapy in the immediate future, early detection also can identify potential candidates for research and clinical trials for therapies in development that target individuals in the earlier stages of their cognitive decline and disease.

Emerging evidence also suggests that early detection of MCI may provide an economic benefit to healthcare systems. Although this has been investigated less extensively in individuals with MCI than in individuals with dementia, published literature suggests that the financial burden associated with caring for MCI patients is significant and that routine cognitive assessment may be cost effective (12-16). Tong et al. investigated screening for MCI and dementia by PCPs in England and reported that PCP use of the Mini-Mental State Examination (MMSE), 6-Item Cognitive Impairment Test and the General Practitioner Assessment of Cognition (GPCOG) led to more quality-adjusted life-years (QALYs) than informal PCP assessment alone (i.e., observing individual cognitive ability) (12). While additional research is needed to further understand the economic benefits of early detection of $\mathrm{MCI}$, existing literature suggests that healthcare systems may derive significant benefits from implementing early detection practices.

\section{Drawbacks of Early Detection of MCI}

While early detection of MCI offers many positive benefits, even in the absence of a disease-modifying therapy, we acknowledge that early detection efforts may not be universally beneficial. For example, false negatives may provide subjects with false reassurance that their cognitive function has not declined, thereby preventing them from seeking further care. Similarly, false positives may create undue stress for impacted individuals and their families. These potential drawbacks underscore the urgent need for an accurate assessment, with sensitivity and specificity sufficient to minimize the detrimental impact of an incorrect result. In the context of an accurate identification, $\mathrm{MCI}$ individuals and their families likely will experience distress upon learning of cognitive impairment. Anecdotally, this panel noted that $\mathrm{MCI}$ individuals might react to this distress by distancing themselves from their physician and/or the healthcare system in response to the societal stigma that exists for individuals with a known cognitive impairment.

Importantly, implementing widespread evaluation of $\mathrm{MCI}$ in the primary care setting may require significant time and resources, representing a burden that may be untenable for all PCPs. Similarly, as routine wellness exams tend to last fewer than twenty minutes, devoting time to cognitive assessment may limit time spent addressing other health concerns. Limited time in PCP visits is likely to be a particularly pressing issue for the 
care of geriatric individuals, who are more likely to have cognitive performance issues but also often have more morbidities and preventative health needs that must be addressed during PCP visits. Additionally, expanding cognitive testing may create an administrative burden for medical personnel, although a digital tool can help minimize this impact. Finally, widespread cognitive assessment is likely to increase the burden on specialists, as greater identification of primary care patients with MCI likely will translate to more referrals to specialists for confirmatory diagnosis. However, if the quality of cognitive assessment in a primary care setting can be improved, this may help identify patients in whom a referral is appropriate, limiting referrals for patients with only a subjective memory complaint (i.e., a self-reported loss of memory performance without objective cognitive decline). Subjective memory complaint (SMC) represents a condition at-risk for $\mathrm{AD}(5,17-21)$. Moreover, SMC may underlie the beginning of the AD clinical continuum $(5,17-21)$, Therefore, it will be even more imperative to provide physicians with the proper tools for timely detection of $\mathrm{AD}$ to allow initiation of appropriate care pathways.

\section{Summary of previous guidelines and consensus statements}

Numerous consensus statements and clinical guidelines have been published in the past to provide perspective and expert guidance on defining and detecting MCI through cognitive testing $(3,22)$. Previous guidelines have summarized the circumstances when cognitive testing becomes appropriate, examined the current testing landscape for MCI detection, and identified the challenges and uncertainties around the detection of MCI. However, an expert consensus with an updated view on the field of cognitive neuroscience in light of novel testing modalities that are now practical due to consumer digital technology, such as smartphone applications, online games and questionnaires, etc., does not currently exist in the literature. Increasing adoption of consumer digital technology and digital fluency, even among older adults, will allow for novel testing modalities that will improve whole patient care and pave the way for potential novel therapies for AD.

A controversy remains in the field of cognitive neuroscience on the appropriate frequency of cognitive testing, as advocates see the benefit of widespread use, while others are proponents of limited and targeted use of cognitive testing. In the U.S., the 2009 Affordable Care Act (ACA) federal legislature mandated an annual cognitive assessment to be conducted during Medicare Annual Wellness visits for seniors $(7,23)$. However, the ACA did not mandate or provide any guidance on what type of testing should be used to meet the ACA requirements, leaving this decision to the discretion of the clinical community. In the wake of the ACA mandate, the controversy on the appropriateness of cognitive testing has continued. In 2014, the United States Preventive Services Task Force concluded that the available evidence was insufficient to assess the benefits (e.g., potential lifestyle interventions and better patient management) and drawbacks of screening for cognitive impairment (e.g., false positives and negatives, patient suspicion and alienation from physicians, etc.) and therefore, could not recommend universal screening (24). However, due to the ACA mandate, there was still a clear need for a consensus from the field on best practices for the development, validation, and use of cognitive testing. This view has been validated by the National Institute on Aging and the Alzheimer's Association AD Framework and the 2015 working group of the International Association of Gerontology and Geriatrics (IAGG) which concluded that early identification of $\mathrm{MCI}$ is essential to improving cognitive performance in older adults (25). The IAGG working group found that benefits can be derived from better management of the treatable components of cognitive impairment and lifestyle interventions that may slow cognitive decline (25). Finally, a 2017 Edinburgh consensus group focused on the implications of diseasemodifying treatments for $\mathrm{AD}$ emphasized the crucial importance of identifying early cognitive impairment, given that therapies will likely be most efficacious early in cognitive decline (26).

In addition to assessing the scenarios in which testing is appropriate, select organizations have published recommendations and advisories on preferred methods for MCI detection. As noted above, the ACA declined to recommend a specific methodology for cognitive assessment in the U.S. given that noconsensus has been reached on a universally accepted screening methodology, and formal guidance has yet to be issued on this topic by federal health authorities (e.g., Centers for Medicare \& Medicaid Services, (CMS)).

In response to the "call to arms" that the ACA mandate represents, an Alzheimer's Association working group outlined specific recommendations for the detection of cognitive impairment in the primary care setting. The expert consensus highlighted that in other countries, such as Canada, the national consensus guidelines have detailed primary care as preferred site for evaluation (https: / / alzheimer.ca/ sites/default / files / files / national / for-hcp/for_hcp_recos_cccdtd4_en.pdf). Moreover, the group recommended that both structured (e.g., use of a formal cognitive test) and unstructured (e.g., informal physician questions about memory) cognitive assessments should be utilized for testing and tracking cognitive function by PCPs (23). A 2018 clinical practices guideline from the American Academy of Neurology noted that relying on subjective cognitive complaints alone is an insufficient assessment criterion for MCI because of the significant potential for over or underidentification (27). The guideline instead recommended that physicians use a validated tool for cognitive 
assessment and solicit patient history along with informant input (27). The 2015 IAGG working group also recommended utilizing both patient and informant assessments of cognitive function along with physician testing to evaluate subjects for early cognitive impairment (25). The group urged the use of validated tests that take only three to seven minutes to conduct, limiting the time burden for patients and providers. Similarly, the Gerontological Society of America workgroup recommended screening tests that take five or fewer minutes to administer, are free of charge, assess multiple cognitive domains, and are validated in a communitybased sample (25). Unfortunately, despite this past guidance, specific and up-to-date recommendations grounded in currently available tools do not yet exist, potentially resulting in uncertainty among PCPs about how best to detect MCI.

In addition to considering when testing is appropriate and what battery of tests is most appropriate, previous groups have also elaborated on how an optimal care pathway may be achieved for early detection of cognitive impairment. This is a critical unmet need in the field, as the uncertainty toward the methodology of assessing for cognitive impairment is compounded by the lack of consensus on what physicians should do in the event of a positive result. Uncertainty about an assessment returns a positive result may de-motivate PCPs from broaching the topic of cognitive impairment or testing with their patients in the first place. In contrast to this PCP-directed recommendation, the 2015 Edinburgh Consensus working group noted that the UK healthcare system would be unable to accommodate the strain on PCPs and specialists if a disease-modifying therapy becomes available, noting that the current role of the $\mathrm{PCP}$ in controlling patient access to specialists is unclear (26). This group emphasized that restructuring cognitive healthcare to allow patients to receive care across disciplines may optimize efficiency and improve patient care (26).

Similarly, despite published insight on select aspects of an "ideal" cognitive assessment (25), it remains unclear which specific tools, particularly digital assessments, are best suited for widespread use and how new assessments could be improved to allow higher detection rates of MCI. This panel sought to provide further clarity on technologies that can improve early detection of MCI, including noting how potential modification or validation of existing tools could contribute to enhanced patient care.

\section{Anticipated changes to the MCI landscape}

The late-stage development of some compounds with a putative disease-modifying effect support optimism that a disease-modifying therapy may become available within the next $3-5$ years (28). Among these Phase 3 agents, monoclonal antibodies targeting amyloid- $\beta$ have recently gaining momentum. Several clinical studies indicate that targeting the early phases of $\mathrm{AD}$, including MCI or even preclinical populations, can increase the likelihood of clinical success (28). This recent focus on early intervention in cognitive decline underscores the value of identification of $\mathrm{MCI}$ before the onset of more severe cognitive decline to maximize the potential for intervention and minimize the personal, clinical, and economic costs of cognitive decline.

Approval of a novel therapy for MCI-AD is expected to dramatically increase both patient and physician involvement in cognitive screening. In the absence of proactive preparation, demand for cognitive assessment will likely present a significant strain on global healthcare systems around the world, with PCPs shouldering a significant portion of the burden. Specialists are also likely to be strained by a large volume of referrals, likely including a minority of patients with MCI-AD and a majority of individuals with MCI due to other etiologies, or even intact cognition (e.g., misdiagnosed or "worried well" individuals with an SMC). It is in the best interest of patients, physicians, and healthcare systems to implement large-scale cognitive screening and to develop and refine technological solutions that can optimize early and accurate detection of MCI and MCI-AD. Going hand in hand with improved detection will also be better patient-physician alignment on the appropriate care pathways once MCI-AD is observed. For example, prior studies evaluating the impact of cholinesterase inhibitors on cognitive symptoms in patients with mild - moderate AD suggest rivastigmine and galantamine may provide statistically significant symptomatic benefits in patients treated earlier in the disease trajectory, which were not achieved for patients in whom treatment was initiated later in the disease course $(29,31)$. Though clinical data to support early initiation of cholinesterase inhibitors in individuals with $\mathrm{MCI}$ at risk of developing $\mathrm{AD}$ is mixed $(27,29,32)$, data from clinical trials investigating anti amyloid- $\beta$ drugs have further supported the importance of early diagnosis and treatment in AD.

\section{Conclusion}

Current neuroscientific discoveries point toward a compelling need to optimize and harmonize clinical protocols for the timely and accurate detection and diagnosis of MCI-AD, also in light of late-stage clinical development of disease-modifying therapies. In subsequent manuscripts, we will outline potential methods to enhance $\mathrm{MCI}$ detection in individuals at risk and across clinical and non-clinical settings, focusing on cognitive, functional, and interview-based approaches. However, we acknowledge the potential for the detection of AD pathology, regardless to the clinical stage, to undergo a substantial change due to technological advances in the future, including blood-based biomarker assays to detect $\mathrm{AD}$. In this case, it may become necessary 
to compare the utility of various screening modalities in parallel through subsequent clinical trials.

The present working group has identified actionable methods to improve cognitive and functional assessment tools, recognizing thatreassessing the optimal care pathway upon the availability of a blood-based biomarkers test with high clinical utility will be necessary for screening and diagnostic purposes. Consequently, blood-based biomarkers should be integrated into the design of studies evaluating the accuracy of cognitive and functional algorithms to detect MCI-AD. Additionally, both biomarkers and cognitive algorithms remain critical components of inclusion criteria and endpoints in clinical trials designed to evaluate MCI-AD therapies.

In this suite of publications, we hope to promote thoughtful but dramatic changes to the existing management plan for adults with MCI, inclusive of cognitive screening for the early detection of MCI. In subsequent articles, we will provide guidance for designing and validating cognitive assessment tools to enhance their real-world utility, which represents a "call to action" for our colleagues in the cognitive evaluation field.

In summary, this task force seeks to support MCI diagnosis and detection of underlying pathophysiology as a public health imperative, in parallel with other major organizations such as the World Health Organization (WHO) and the Alzheimer's Association, in order to improve clinical outcomes for aging individuals - not just in preparation for a novel AD therapy but also in the current context of this field.

Acknowledgements and funding: Medical writing support, under the direction of the authors, was provided by ClearView Healthcare Partners, LLC, funded by Eisai Inc., in accordance with Good Publication Practice (GPP3) guidelines.

Disclosures: MB is affiliated with the Research Center and Memory Clinic Fundació ACE, Institut Català de Neurociències Aplicades, Universitat Internacional de Catalunya, Barcelona, Spain; and with the Networking Research Center on Neurodegenerative Diseases (CIBERNED), Instituto de Salud Carlos III, Spain. Private research funding sources include Grifols SA; Caixabank S.A.; Life Molecular Imaging; Araclon Biotech; Laboratorios Echevarne; Festival Castell Paralada; Bonpreu/Esclat; and Famila Carbó. Public grants include those from Instituto de Salud Carlos III. Ministerio de Salud. Gobierno de España; Dirección General de Farmacia. Ministerio de Salud. Gobierno de España; and European Commission:H2020 program, Innovative Medicine Initiative (IMI-2); and ERA-NET NEURON program, European Marie Sklodowska Curie. Advisory work includes that for Araclon Biotech, Biogen, Bioibérica, Eisai, Grifols, Lilly, Merck, Nutricia, Roche, Oryzon, Schwabe Farma, Servier, and Kyowa Kirin. PMD has received research grants (through Duke University) from Avid, Lilly, Neuronetrix, Avanir, Bauch, Alzheimer's Drug Discovery Foundation, Cure Alzheimer's Fund, Wrenn Trust, DOD, ONR, and NIH. PMD has received speaking or advisory fees from Anthrotronix, Neuroptix, Genomind, Clearview, Cognicity, Nutricia, Living Media, Verily, RBC, Brain Canada, and CEOs Against Alzheimers. PMD owns shares in Muses Labs, Anthrotronix, Evidation Health, Turtle Shell Technologies and Advera Health Analytics whose products are not discussed here. He has received travel support from World Economic Forum, CCABH and Canaan Ventures. PMD served/serves on the board of Baycrest, AHEL, TLLF and TGHF. PMD is a co-inventor (through Duke) on patents relating to dementia biomarkers and therapies. BD is affiliated with the Institute of Memory and Alzheimer's Disease (IM2A), Department of Neurology, Salpêtrière Hospital, AP-HP, Sorbonne-Université, Paris, France. JI is affiliated as an Assistant Professor-Adjunct (Group1) with the Department of Family Practice at Queen's University. She was also a panelist for Hoffman-La Roche Limited, Ottawa, on an Alzheimer's Disease Panel. She is involved with the Canadian Consortium on Neurodegeneration in Aging (CCNA), Extension Study as a Co- Investigator and Research Coordinator. AI received lecture fees from Eisai, Janssen, Otsuka, Eli Lilly, MSD, Chugai-Roche, Daiichi-Sankyo, Alnylam, Takeda, UCB, Ono,
Integra Japan, IQVIA, Fuji Rebio, Biogen, and advisory fees from Janssen during the past three years. AP reports personal fees from Acadia Pharmaceuticals, Functional Neuromodulation, Neurim Pharmaceuticals, Grifols, Eisai, BioXcel, Tetra Discovery Partners, and Merck; grants from AstraZeneca, Avanir, Biogen, Biohaven, Eisai, Eli Lilly, Janssen, Genentech/Roche, Novartis, Merck, as well as funding from NIA, NIMH, DOD. KLP receives grant funding from the National Institute on Aging, the National Institute of Neurological Disorders and Stroke, the Global Brain Health Institute, and Quest Diagnostics. BV has consultancy and research grants from Roche, Biogen, EISAI, Nestle, Lilly, Cerecin, and Merck. $\mathrm{HH}$ is an employee of Eisai Inc. and serves as Senior Associate Editor for the Journal Alzheimer's \& Dementia; during the past three years he had received lecture fees from Servier, Biogen and Roche, research grants from Pfizer, Avid, and MSD Avenir (paid to the institution), travel funding from Eisai, Functional Neuromodulation, Axovant, Eli Lilly and company, Takeda and Zinfandel, GE-Healthcare and Oryzon Genomics, consultancy fees from Qynapse, Jung Diagnostics, Cytox Ltd., Axovant, Anavex, Takeda and Zinfandel, GE Healthcare, Oryzon Genomics, and Functional Neuromodulation, and participated in scientific advisory boards of Functional Neuromodulation, Axovant, Eisai, Eli Lilly and company, Cytox Ltd., GE Healthcare, Takeda and Zinfandel, Oryzon Genomics and Roche Diagnostics. He is co-inventor in the following patents as a scientific expert and has received no royalties: - In Vitro Multiparameter Determination Method for the Diagnosis and Early Diagnosis of Neurodegenerative Disorders Patent Number: 8916388; • In Vitro Procedure for Diagnosis and Early Diagnosis of Neurodegenerative Diseases Patent Number: 8298784; • Neurodegenerative Markers for Psychiatric Conditions Publication Number: 20120196300; • In Vitro Multiparameter Determination Method for The Diagnosis and Early Diagnosis of Neurodegenerative Disorders Publication Number: 20100062463; • In Vitro Method for The Diagnosis and Early Diagnosis of Neurodegenerative Disorders Publication Number: 20100035286; - In Vitro Procedure for Diagnosis and Early Diagnosis of Neurodegenerative Diseases Publication Number: 20090263822; - In Vitro Method for The Diagnosis of Neurodegenerative Diseases Patent Number: 7547553; - CSF Diagnostic in Vitro Method for Diagnosis of Dementias and Neuroinflammatory Diseases Publication Number: 20080206797; • In Vitro Method for The Diagnosis of Neurodegenerative Diseases Publication Number: 20080199966; • Neurodegenerative Markers for Psychiatric Conditions Publication Number: 20080131921. AV is an employee of Eisai Inc. and received lecture honoraria from Roche, MagQu LLC, and Servier.

Open Access: This article is distributed under the terms of the Creative Commons Attribution 4.0 International License (http:// creativecommons.org/ licenses/by/4.0/), which permits use, duplication, adaptation, distribution and reproduction in any medium or format, as long as you give appropriate credit to the original author(s) and the source, provide a link to the Creative Commons license and indicate if changes were made.

\section{References}

1. Global, regional, and national under-5 mortality, adult mortality, age-specific mortality, and life expectancy, 1970-2016: a systematic analysis for the Global Burden of Disease Study 2016. Lancet Lond Engl 2017;390:1084-1150

2. Global, regional, and national disability-adjusted life-years (DALYs) for 359 diseases and injuries and healthy life expectancy (HALE) for 195 countries and territories, 1990-2017: a systematic analysis for the Global Burden of Disease Study 2017. Lancet Lond Engl 2018;392:1859-1922

3. Albert MS, DeKosky ST, Dickson D, et al. The diagnosis of mild cognitive impairment due to Alzheimer's disease: recommendations from the National Institute on Aging-Alzheimer's Association workgroups on diagnostic guidelines for Alzheimer's disease. Alzheimers Dement J Alzheimers Assoc 2011;7:270-279

4. Petersen RC, Caracciolo B, Brayne C, Gauthier S, Jelic V, Fratiglioni L. Mild cognitive impairment: a concept in evolution. J Intern Med 2014;275:214-228

5. Jack CR, Bennett DA, Blennow K, et al. NIA-AA Research Framework: Toward a biological definition of Alzheimer's disease. Alzheimers Dement J Alzheimers Assoc 2018;14:535-562

6. Hampel H, O’Bryant SE, Molinuevo JL, et al. Blood-based biomarkers for Alzheimer disease: mapping the road to the clinic. Nat Rev Neurol 2018;14:639-652

7. 2019 Alzheimer's disease facts and figures. Alzheimers Dement J Alzheimers Assoc 2019;15:321-387

8. Nasreddine ZS, Phillips NA, Bédirian V, et al. The Montreal Cognitive Assessment, MoCA: a brief screening tool for mild cognitive impairment. J Am Geriatr Soc 2005;53:695-699

9. De Roeck EE, De Deyn PP, Dierckx E, Engelborghs S. Brief cognitive screening instruments for early detection of Alzheimer's disease: a systematic review. Alzheimers Res Ther 2019;11:21

10. Borson S, Frank L, Bayley PJ, et al. Improving dementia care: The role of screening and detection of cognitive impairment. Alzheimers Dement J Alzheimers Assoc 2013;9:151-159

11. Morley JE. Anticholinergic medications and cognition. J Am Med Dir Assoc 2011;12:543-543.e1 
12. Tong T, Thokala P, McMillan B, Ghosh R, Brazier J. Cost effectiveness of using cognitive screening tests for detecting dementia and mild cognitive impairment in primary care: Cost effectiveness of cognitive screening tests in primary care. Int J Geriatr Psychiatry 2017;32:1392-1400

13. Ton T, DeLeire T, May S, et al. Assessing the Financial Burden Associated with Mild Cognitive Impairment. 2015

14. Ton TGN, DeLeire T, May SG, et al. The financial burden and health care utilization patterns associated with amnestic mild cognitive impairment. Alzheimers Dement J Alzheimers Assoc 2017;13:217-224

15. Pennington M, Gomes M, Chrysanthaki T, et al. The cost of diagnosis and early support in patients with cognitive decline. Int J Geriatr Psychiatry 2018;33:5-13

16. Gomes M, Pennington M, Black N, Smith S. Cost-effectiveness analysis of English memory assessment services 2 years after first consultation for patients with dementia. Int J Geriatr Psychiatry 2019;34:439-446

17. Choe YM, Byun MS, Lee JH, Sohn BK, Lee DY, Kim JW. Subjective memory complaint as a useful tool for the early detection of Alzheimer's disease. Neuropsychiatr Dis Treat 2018;14:2451-2460

18. Brailean A, Steptoe A, Batty GD, Zaninotto P, Llewellyn DJ. Are subjective memory complaints indicative of objective cognitive decline or depressive symptoms? Findings from the English Longitudinal Study of Ageing. J Psychiatr Res 2019;110:143-151

19. Wanrooij LL van, Richard E, Jongstra S, Charante EPM van, Gool WA van Associations of Subjective Memory Complaints and Simple Memory Task Scores With Future Dementia in the Primary Care Setting. Ann Fam Med 2019;17:412-418

20. Buckley RF, Maruff P, Ames D, et al. Subjective memory decline predicts greater rates of clinical progression in preclinical Alzheimer's disease. Alzheimers Dement J Alzheimers Assoc 2016;12:796-804

21. van Harten AC, Mielke MM, Swenson-Dravis DM, et al. Subjective cognitive decline and risk of MCI: The Mayo Clinic Study of Aging. Neurology 2018;91:e300-e312
22. Gauthier S, Reisberg B, Zaudig M, et al. Mild cognitive impairment. Lancet Lond Engl 2006;367:1262-1270

23. Cordell CB, Borson S, Boustani M, et al. Alzheimer's Association recommendations for operationalizing the detection of cognitive impairment during the Medicare Annual Wellness Visit in a primary care setting. Alzheimers Dement 2013;9:141-150

24. Moyer VA. Screening for Cognitive Impairment in Older Adults: U.S Preventive Services Task Force Recommendation Statement. Ann Intern Med 2014;160:791

25. Morley JE, Morris JC, Berg-Weger M, et al. Brain Health: The Importance of Recognizing Cognitive Impairment: An IAGG Consensus Conference. J Am Med Dir Assoc 2015;16:731-739

26. Ritchie CW, Russ TC, Banerjee S, et al. The Edinburgh Consensus: preparing for the advent of disease-modifying therapies for Alzheimer's disease. Alzheimers Res Ther 2017;9:85

27. Petersen RC, Lopez O, Armstrong MJ, et al. Practice guideline update summary: Mild cognitive impairment: Report of the Guideline Development, Dissemination, and Implementation Subcommittee of the American Academy of Neurology. Neurology 2018;90:126-135

28. Cummings J, Lee G, Ritter A, Sabbagh M, Zhong K. Alzheimer's disease drug development pipeline: 2019. Alzheimers Dement N Y N 2019;5:272-293

29. Hampel H, Mesulam M-M, Cuello AC, et al. The cholinergic system in the pathophysiology and treatment of Alzheimer's disease. Brain J Neurol 2018;141:1917-1933

30. Farlow MR, Cyrus PA. Metrifonate therapy in Alzheimer's disease: a pooled analysis of four randomized, double-blind, placebo-controlled trials. Dement Geriatr Cogn Disord 2000;11:202-211

31. Raskind MA, Peskind ER, Wessel T, Yuan W. Galantamine in AD: A 6-month randomized, placebo-controlled trial with a 6-month extension. The Galantamine USA-1 Study Group. Neurology 2000;54:2261-2268

32. Russ TC, Morling JR. Cholinesterase inhibitors for mild cognitive impairment. Cochrane Database Syst Rev 2012;CD009132 